\title{
An Unusual Presentation of Rhabdomyolysis in a Newly Diagnosed Sjögren's Syndrome Associated Polymyositis
}

\author{
Shanti VIRUPANNAVAR, Suncica VOLKOV \\ Department of Rheumatology, University of Illinois at Chicago, Chicago, United States
}

\begin{abstract}
Rhabdomyolysis is a serious and potentially life-threatening syndrome due to rapid breakdown of skeletal muscle fibers. Acquired and genetic causes should be investigated when patients present with rhabdomyolysis. In this article, we report a patient of polymyositis associated with Sjögren's syndrome, inducing rhabdomyolysis.

Keywords: Myositis; polymyositis; rhabdomyolysis; Sjögren's syndrome.
\end{abstract}

Rhabdomyolysis is a serious and potentially life-threatening syndrome, caused by rapid breakdown of skeletal muscle fibers, resulting in leakage of toxic muscle cell content into systemic circulation. ${ }^{1,2}$ Approximately 26,000 cases of rhabdomyolysis are reported in the United States per year. ${ }^{2}$ The clinical presentation of rhabdomyolysis is quite varied, ranging from asymptomatic elevation of serum creatinine kinase $(\mathrm{CK})$ to cardiac arrhythmia, acute renal failure, and disseminated intravascular coagulation. ${ }^{2}$ Acquired and genetic causes should be investigated in case of rhabdomyolysis. The most common acquired causes are substance abuse, medications, and trauma. ${ }^{2,3}$ Other less frequent causes include infection, electrolyte disturbances, endocrine disorders, extreme physical exertion, body temperature changes, and metabolic myopathies. ${ }^{2}$

A literature review revealed only three reported cases of polymyositis associated rhabdomyolysis. ${ }^{3-5}$ To our knowledge, there are no documented cases of polymyositis associated with Sjögren's syndrome, inducing rhabdomyolysis.
In this article, we report a patient who presented with acute onset of myalgia and pigmenturia and was later diagnosed with polymyositis associated with Sjögren's syndrome.

\section{CASE REPORT}

A 52-year-old African American male patient with a history of hypertension presented to hospital with complaints of blood in his urine and sudden onset of bilateral buttock, thigh, and shoulder muscle pains for the last week. Patient denied any upper respiratory symptoms, usage of medications, illicit drugs, supplements, or vitamins, recent prolonged immobilization, trauma, alcohol use or strenuous exercise. A written informed consent was obtained from the patient.

Physical examination was unremarkable except for mild parotid and lacrimal gland enlargement. Pertinent laboratory tests at admission included CK elevation of $136.461 \mathrm{U} / \mathrm{L}$, and marked aspartate and alanine aminotransferase elevations [557 U/L

Received: August 17, 2015 Accepted: February 10, 2016 Published online: April 20, 2016

Correspondence: Shanti Virupannavar, MD. Department of Rheumatology, University of Illinois at Chicago, 60605 Chicago, United States.

Tel: 312-413-9310 e-mail: shantivirup@gmail.com

o2016 Turkish League Against Rheumatism. All rights reserved. 
(10-40) and $106 \mathrm{U} / \mathrm{L}$ (10-50), respectively]. Urine was positive for myoglobin. The patient had extensive negative work-up for various causes of rhabdomyolysis including thyroid stimulating hormone, human immunodeficiency virus, alcohol level, hemoglobin A1c, drug screen, viral panel, and electrolytes. He had a computed axial tomography scan of his abdomen/pelvis showing no obstructive uropathy.

Despite treatment with aggressive hydration, CK further increased to $169.158 \mathrm{U} / \mathrm{L}$ with no improvement in symptoms over the next two days. Rheumatology was consulted for work-up of inflammatory myopathy as a cause of his rhabdomyolysis.

The patient had positive antinuclear antibody, with titer 1:1280 in a speckled immunofluorescence pattern and positive Sjögren's syndrome antibody. The erythrocyte sedimentation rate was mildly elevated at $35 \mathrm{~mm} /$ hour and anti Jo-1 antibody was negative. These findings, along with the physical exam findings of lacrimal and parotid glands enlargement, were consistent with the Sjögren's syndrome. The magnetic resonance imaging of bilateral thighs showed symmetric muscle edema of the thigh muscles (Figure 1). There was also moderate symmetric edema of the tensor fascia lata and gluteus maximus muscles. Given the symmetric muscle involvement and positive antinuclear antibody markers, rhabdomyolysis was deemed to be related to an

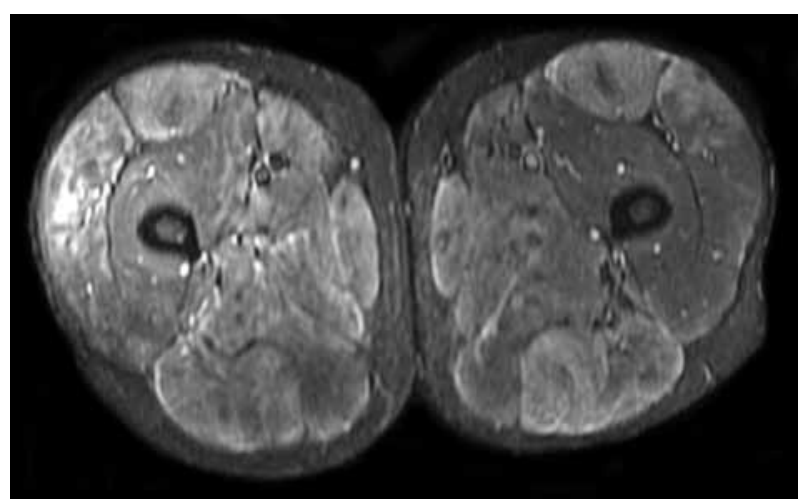

Figure 1. Axial $T_{1}$ short tau inversion recovery magnetic resonance imaging of bilateral lower extremities depicting moderate edema in bilateral thigh muscles. Edema is slightly more prominent in right thigh muscles, with relative sparing of the bilateral vastus intermedius and semimembranosus muscles. There is symmetric edema of adductor and gluteus maximus and tensor fascia lata muscles. inflammatory myopathy associated with Sjögren's syndrome.

A biopsy of quadriceps muscle demonstrated active myopathic changes and perivascular and endomysial mononuclear focal inflammatory infiltrate (Figure 2). No rimmed vacuoles or abnormal accumulation of lipids or mitochondria were identified. The immunohistochemical staining for major histocompatibility complex class 1 was equivocal. Histochemical stains showed normal myophosphorylase, phosphofructokinase and myoadenylate deaminase, succinate dehydrogenase, nicotinamide adenine dinucleotide + hydrogen and cytochrome oxidase activity. These findings confirmed the diagnosis of polymyositis.

Patient was treated with $60 \mathrm{mg}$ of prednisone per day for five days, with subsequent taper. $\mathrm{He}$ had a marked, rapid improvement of $\mathrm{CK}$ and symptoms upon initiation of steroids. At follow-up, less than four weeks after discharge, patient was tapered down to prednisone of $30 \mathrm{mg}$ per day and had complete resolution of symptoms. Azathioprine, a steroid sparing agent, was administered while tapering prednisone.

\section{DISCUSSION}

Polymyositis is an idiopathic inflammatory myopathy characterized by muscle weakness,

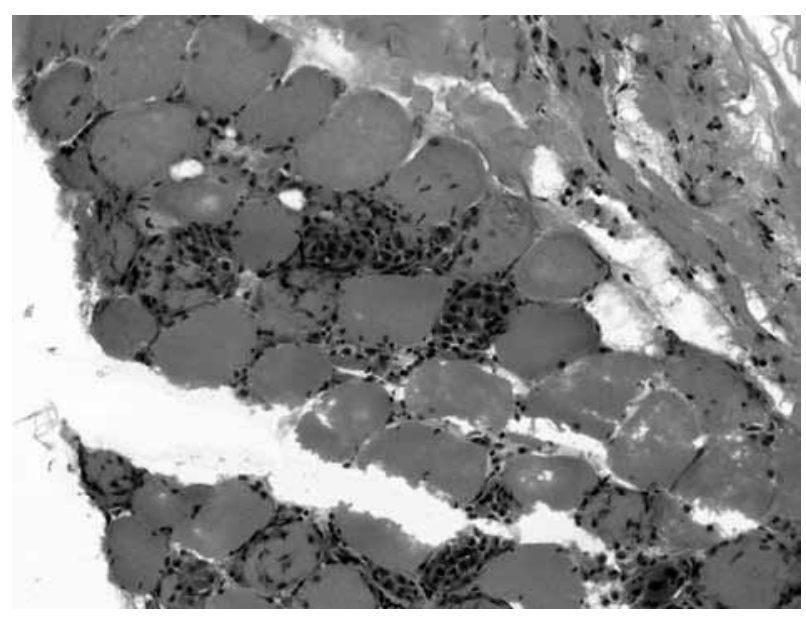

Figure 2. Muscle biopsy of quadriceps muscle showing active myopathic changes and perivascular and endomysial mononuclear focal inflammatory infiltrate. No rimmed vacuoles or abnormal accumulation of lipids or mitochondria were identified. 
typically proximal and symmetric, elevation in muscle enzymes, and distinctive findings of electromyogram and muscle biopsy. 6,7 This disease may be associated with infections, sarcoid or connective tissues disease, such as Sjögren's syndrome. ${ }^{6,8-10}$ It is usually responsive to corticosteroids and immunosuppression. ${ }^{7}$ Typical pathologic findings include endomysial mononuclear inflammatory infiltrate, which is CD8+ T cell predominant, major histocompatibility complex class 1 expression, and myofiber necrosis. ${ }^{7}$

Our patient presented with symptoms of rhabdomyolysis including myalgia and myoglobinuria and was subsequently diagnosed with polymyositis, based on the features of magnetic resonance imaging and muscle biopsy. Polymyositis has only rarely been described as a cause for rhabdomyolysis. ${ }^{3-5}$ Typically, polymyositis is of gradual onset, with symptoms progressing over several months, rarely presenting as an acute episode as in our patient. To our knowledge, there are no documented cases of polymyositis associated with Sjögren's syndrome, inducing rhabdomyolysis.

Though cases of inflammatory myopathy causing myoglobinuria and rhabdomyolysis are rare, it is important to have a high index of suspicion to avoid end organ damage. One should consider this diagnosis in patients who do not respond appropriately to supportive treatment and hydration, and have sustained myoglobinuria. It is also essential to take a good history and look for clues of autoimmune etiologies, such as Sjögren's syndrome.

\section{Declaration of conflicting interests}

The authors declared no conflicts of interest with respect to the authorship and/or publication of this article.

\section{Funding}

The authors received no financial support for the research and/or authorship of this article.

\section{REFERENCES}

1. Sauret JM, Marinides G, Wang GK. Rhabdomyolysis. Am Fam Physician 2002;65:907-12.

2. Zutt R, van der Kooi AJ, Linthorst GE, Wanders de Visser M. Rhabdomyolysis: review of the literature. Neuromuscul Disord 2014;24:651-9.

3. Thakur V, DeSalvo J, McGrath H Jr, Weed S, Garcia C. Case report: polymyositis-induced myoglobinuric acute renal failure. Am J Med Sci 1996;312:85-7.

4. Pirovino M, Neff MS, Sharon E. Myoglobinuria and acute renal failure with acute polymyositis. N Y State J Med 1979;79:764-7.

5. Kim HW, Choi JR, Jang SJ, Chang YS, Bang BK, Park $\mathrm{CW}$. Recurrent rhabdomyolysis and myoglobinuric acute renal failure in a patient with polymyositis. Nephrol Dial Transplant 2005;20:2255-8.

6. Christopher-Stine L, Plotz PH. Myositis: an update on pathogenesis. Curr Opin Rheumatol 2004;16:700-6.

7. Luo YB, Mastaglia FL. Dermatomyositis, polymyositis and immune-mediated necrotising myopathies. Biochim Biophys Acta 2015;1852:622-32.

8. Caccamo DV, Keene CY, Durham J, Peven D. Fulminant rhabdomyolysis in a patient with dermatomyositis. Neurology 1993;43:844-5.

9. Rose MR, Kissel JT, Bickley LS, Griggs RC. Sustained myoglobinuria: the presenting manifestation of dermatomyositis. Neurology 1996;47:119-23.

10. Joshi D, Kumar N, Rai A. Dermatomyositis presenting with rhabdomyolysis and acute renal failure; an uncommon manifestation. Ann Indian Acad Neurol 2009;12:45-7. 